\title{
La tour Eiffel : le premier laboratoire d'aérodynamique en France
}

De la crise de Panama à la crise de trainée

Cet article décrit sommairement

la recherche expérimentale

de Gustave Eiffel, qui a mis

en évidence la forte réduction

de la trainée d'une sphère dans

un écoulement à grande vitesse, accompagnant le changement

occasionné par la transition

de la couche limite du corps

depuis l'état laminaire vers l'état

turbulent (crise de trainée).

Cette découverte, mise en question

initialement par Ludwig Prandtl,

a été rendue possible par la

conception et la réalisation par

Eiffel d'un ensemble de nouvelles

expériences astucieuses et de

techniques innovantes créées

autour de la tour Eiffel.

Un siècle après la découverte

de cet effet, la compréhension

de la dynamique de transition

turbulente intermittente

et bistable ainsi que la description

des structures cohérentes instables

présentes dans cette transition,

restent ouvertes.

José Eduardo Wesfreid (wesfreid@pmmh.espci.fr)

Laboratoire PMMH, ESPCI, 10 rue Vauquelin, 75231 Paris Cedex 05

En 2019, les cent-trente ans de la tour Eiffel ont donné lieu à plusieurs commémorations [1]. En tant que physiciens, nous sommes intéressés par les aspects scientifiques sous-jacents à l'œuvre du grand ingénieur que fut Gustave Eiffel, en particulier par le calcul de la forme de la tour en réaction au vent [2] ainsi que par l'utilisation de cette œuvre monumentale, la première de son temps, pour des études météorologiques [3] et les premières réalisations de la TSF [4]. Mais il existe d'autres aspects, moins connus, qui sont les recherches d'Eiffel sur l'aérodynamique, qu'il a réalisées dans l'enceinte même de la tour.

\section{L'appareil de chute libre guidée}

Déjà en 1892, deux chercheurs, LouisPierre Cailletet et Eugène Colardeau, avec le soutien d'Étienne-Jules Marey et de Gustave Eiffel, réalisèrent une série d'expériences consistant à laisser tomber du deuxième étage de la tour (soit à 115,7 mètres du sol) des objets en chute libre, tenus par un fil fin et léger, en mesurant la vitesse par le déroulement du fil. La résistance de l'air - égale au poids de l'objet en chute libre une fois atteinte sa vitesse limite - est évaluée, ce qui permet d'obtenir un coefficient de proportionnalité entre la vitesse au carré et la section du corps (en général carrée ou circulaire) [5].

En 1893, Eiffel se retire de la vie professionnelle après sa participation malheureuse à la Compagnie du Canal de Panama, secouée par de nombreux scandales politiques et financiers qui aboutirent à diverses poursuites pénales auxquelles il échappa finalement. C'est alors qu'il décide de se consacrer à la recherche en s'intéressant au vent et à la météorologie. Il reprend en 1903 les expériences de chute des corps depuis la tour, et conçoit un appareil plus astucieux permettant une mesure de la vitesse instantanée et de la force sur le corps.

Cet appareil (fig. 1) est constitué par un lourd bâti, portant dans son extrémité les surfaces à tester et glissant le long d'un câble vertical de 115 mètres de hauteur. Le câble, à 21 mètres du sol, est augmenté dans son diamètre (" cône d'arrêt ") afin de freiner l'objet, permettant ainsi de mesurer la variation de la vitesse durant la décélération. Le temps écoulé est enregistré depuis le début de la chute, en même temps que la distance parcourue par l'appareil et la force sur l'objet d'essai. Ces mesures sont effectuées à l'aide d'un diapason et d'un tambour tournant à une vitesse proportionnelle à celle du déplacement sur le câble, et sur lequel un stylet enregistre la force mesurée par les ressorts dynamométriques tenant l'objet.

La vitesse de chute $U$ varie entre 15 et $40 \mathrm{~m} / \mathrm{s}$ (vitesse maximale), ce qui a permis à Eiffel de mesurer de façon précise le coefficient de résistance (défini comme le rapport entre la force de résistance par unité de surface normale au déplacement et la pression dynamique $\rho_{\text {air }} \mathrm{U}^{2} / 2$ ) et sa variation avec la vitesse sur des corps de différentes géométries (sphères, cônes, hémisphères, plaques et disques inclinés). En 1907, il publie ses résultats dans un long mémoire intitulé Recherches expérimentales sur la résistance de l'air [6], ce qui lui assure une rapide communication internationale de ses résultats scientifiques. L'appareil de chute et la tour deviennent ainsi le premier laboratoire aérodynamique français [7]. 

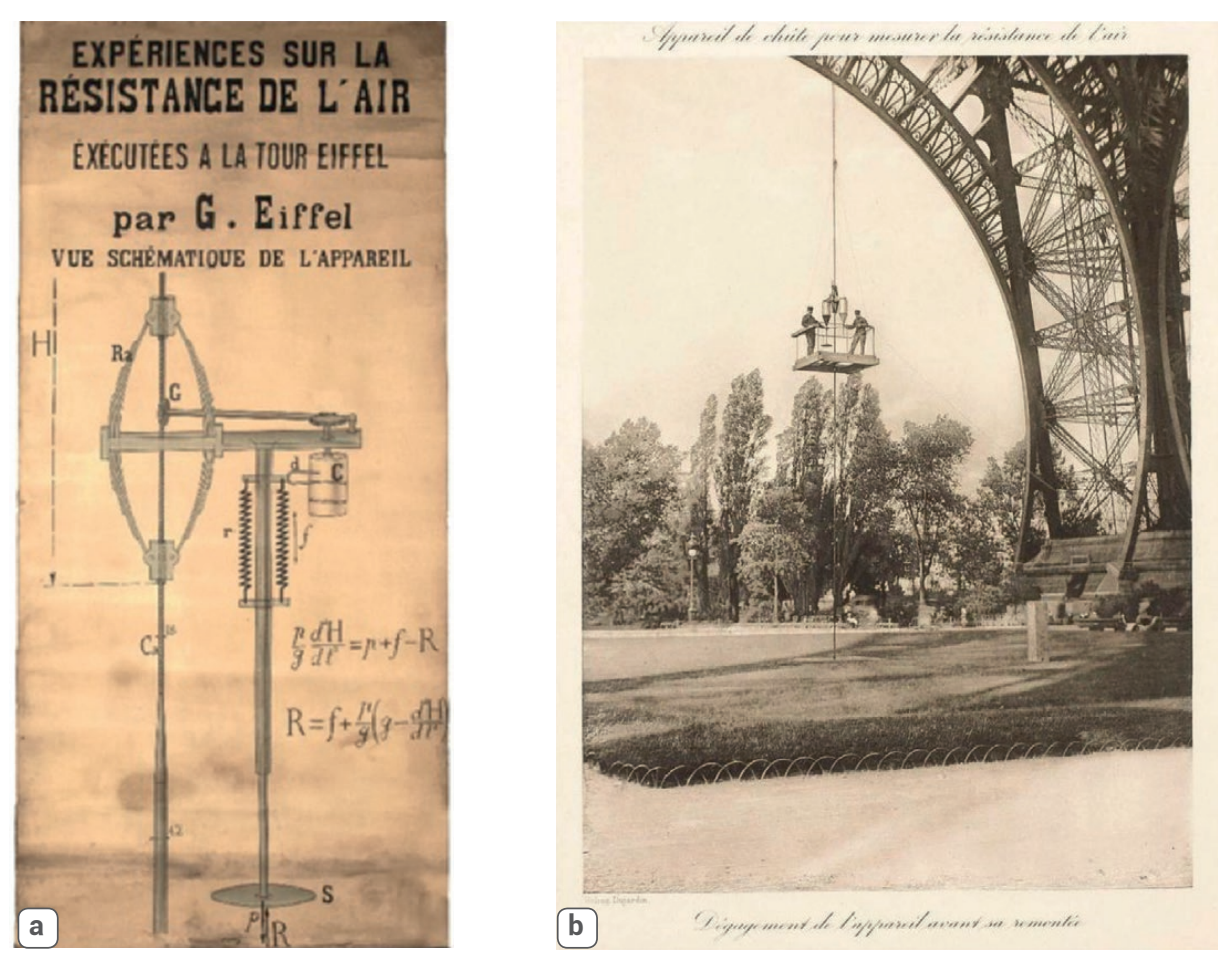

1. Expériences sur la résistance de l'air effectuées à la tour Eiffel.

(a) Vue schématique de l'appareil de chute libre guidée (1903). La vitesse, nulle au départ à $115 \mathrm{~m}$ de hauteur, augmente constamment jusqu'à $40 \mathrm{~m} / \mathrm{s}$ au point de freinage (cône d'arrêt). Le corps (plaque) à tester, placé en $S$, tend les ressorts $r$ lors de sa chute. La résistance de l'air $(R)$ est déduite de la force (tension des ressorts) inscrite par un stylet sur le cylindre tournant $\mathrm{C}$ recouvert de noir de fumée.

(b) Dégagement de l'appareil sur le cône d'arrêt à 21 m du sol avant sa remontée. ouverte ("la méthode du tunnel ») qui permet d'obtenir, dans une section de $1,5 \times 1,5 \mathrm{~m}$, une vitesse de $18 \mathrm{~m} / \mathrm{s}$ par aspiration de l'air dans une chambre, grâce à un moteur de 50 chevaux $(36,8 \mathrm{~kW})$ alimenté par les générateurs électriques de la tour qui font de cette soufflerie la plus puissante du monde (fig. 2a). L'installation est équipée d'une balance aérodynamique imaginée par Eiffel, ainsi que par un appareil destiné à la détermination des centres de poussée (la « girouette »), qui se révèlera très utile pour l'étude de la stabilité des ailes d'avion et en particulier pour observer comment, à partir d'une certaine inclinaison, apparait un brusque déplacement du centre de pression de l'avant vers l'arrière. Ce modèle de "soufflerie Eiffel " sera rapidement adopté par de nombreux laboratoires aérodynamiques dans le monde. Un autre modèle très répandu et performant est celui de Ludwig Prandtl, qui construisit en 1908 dans son laboratoire de Göttingen une soufflerie en circuit fermé. Plus de 5000 essais seront réalisés dans cette installation du Champ de Mars, inaugurée en 1909, puis détruite en 1911 à cause des nuisances sonores et du renouvellement du quartier.

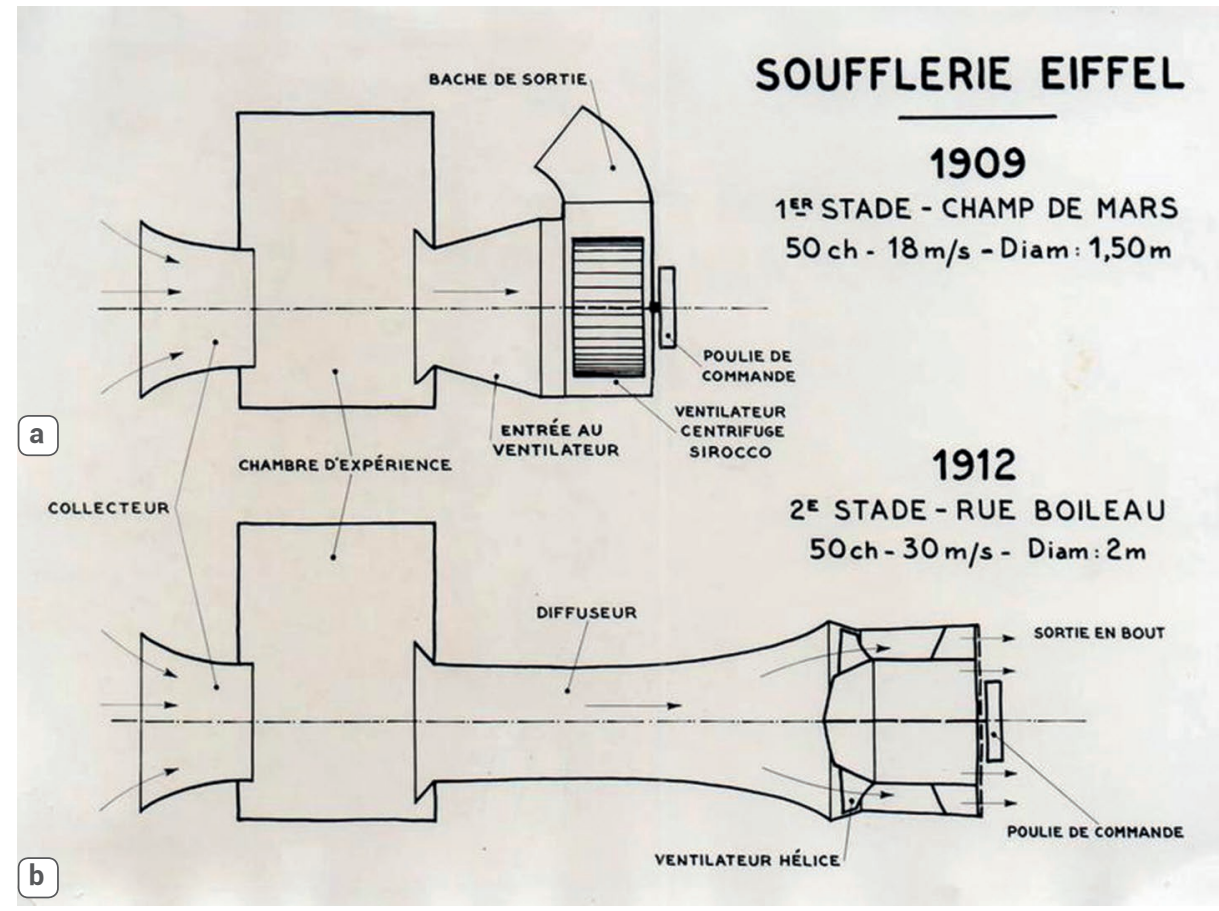

2. Schémas des souffleries Eiffel au Champ de Mars (a) et rue Boileau (b). Le diffuseur est une innovation d'Eiffel qui permet d'améliorer le rendement énergétique des souffleries. 


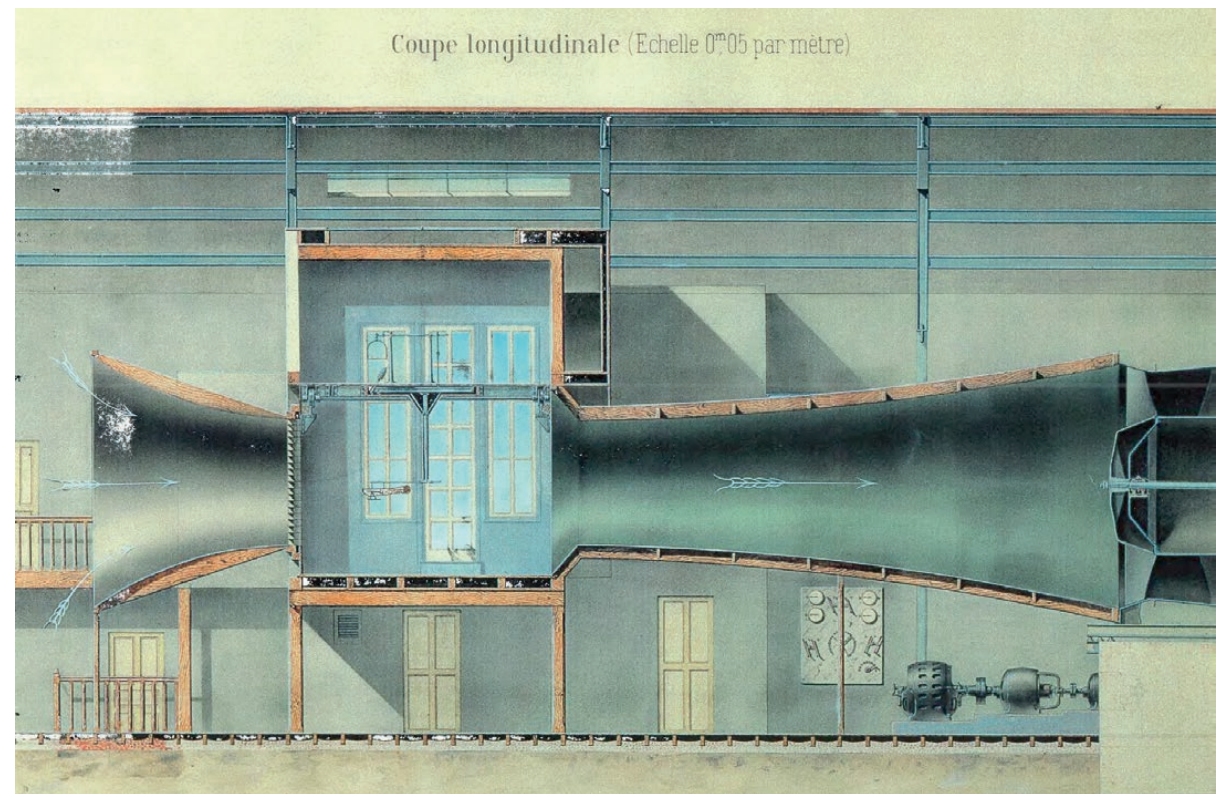

3. Coupe longitudinale de la soufflerie d'Auteuil.

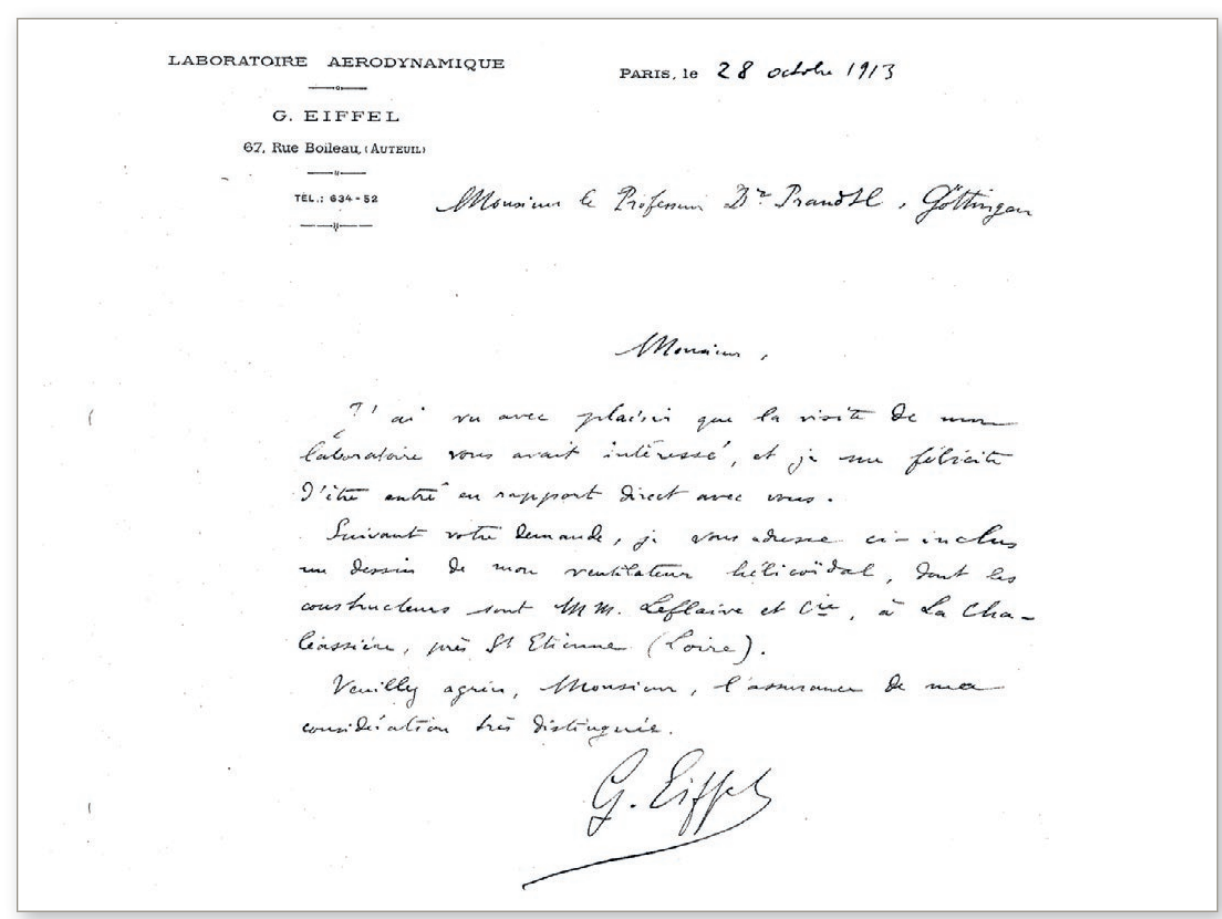

4. Lettre d'Eiffel à Prandtl pour transmettre les plans de son ventilateur.

(Archives GOAR 3684 DLR [10]).
À 79 ans, Gustave Eiffel reconstruit à Auteuil une soufflerie encore opérationnelle de nos jours ${ }^{(a)}$, en élargissant sa veine d'essai à $2 \times 2 \mathrm{~m}$, améliorant ainsi le rendement de l'installation grâce à la conception d'un diffuseur qui lui permit d'atteindre des vitesses de $30 \mathrm{~m} / \mathrm{s}$ (figs. $2 \mathrm{~b}$ et 3 ).

\section{Découverte de l'effet de la « crise de trainée »}

Parmi les milliers de résultats obtenus par Eiffel sur la résistance des objets dans l'air, quelques-uns méritent une attention particulière et motivèrent, en leur temps, une polémique. Il s'agit des expériences sur la résistance des sphères en mouvement à grande vitesse. En 1912, Otto Föppl, chercheur à Göttingen, suspecte dans une publication [8] que les valeurs mesurées par Eiffel du coefficient de résistance des sphères sont entachées d'erreurs "manifestes ", car les mesures effectuées à Göttingen montrent des valeurs deux fois plus grandes. Eiffel répond immédiatement dans une note aux Comptes Rendus [9] que, si pour des vitesses inférieures à une certaine vitesse critique les valeurs trouvées par lui-même correspondent bien à celles mesurées à Göttingen, pour des vitesses plus grandes on trouve l'effet paradoxal que la trainée diminue presque de moitié (fig. 5a), valeurs que l'on ne pouvait obtenir à Göttingen où l'écoulement de la soufflerie était moins rapide qu'à Paris !

Eiffel venait ainsi de découvrir l'effet de la " crise de trainée ", observée sur des corps en mouvement à partir d'une certaine valeur critique de la vitesse. En terme de vitesse adimensionnelle exprimée par le nombre de Reynolds $\mathrm{Re}(\mathrm{Re}=\mathrm{UL} / \mathrm{v}$, où $\mathrm{U}$ et $\mathrm{L}$ sont la vitesse et la taille caractéristiques et $v$ est la viscosité cinématique $\mathrm{du}$ fluide), ce phénomène se manifeste quand Re est de l'ordre de $3 \times 10^{5}$ pour les sphères (fig. 5b).

\section{Références}

1 Par exemple l'Expo des 130 ans, à laquelle notre laboratoire a participé www.toureiffel.paris/fr/actualites/ 130-ans/lexpo-des-130-ans-le-rendezvous-de-lautomne

2• P.D. Weidman, Physics of Fluids, 21 (2009) 067102.

3• A. Angot, J. Phys. Theor. Appl., 9 (1) (1890) 169-177.

4. E. Ducretet, C.R. hebd. séances Acad. Sci. 127 (1898) 713-716.
5. L. Cailletet et E. Colardeau, C.R. hebd. séances Acad. Sci. 115 (1892) 13-19.

6• G. Eiffel, Recherches expérimentales sur la résistance de l'air exécutées à la Tour Eiffel, L. Maretheux, Paris (1907), réédité en 2016 par Hachette Livre BNF.

7• C. Fontanon, dans Histoire de la mécanique appliquée, pp. 57-88, ENS Éditions (1998).

8 0. Föppl, Zeitschrift für Flugtechnik und Motoruftschiffahrt, 3 (1912) 118-121.
9• G. Eiffel, C.R. hebd. séances Acad. Sci. 155 (1912) 1597-1599.

10 M. Eckert, Ludwig Prandtl: A Life for Fluid Mechanics and Aeronautical Research, Springer (2019).

11 S.S. Tiwari et al., Powder Technology, 365 (2020) 215-243.

12 • M. Geier, A. Pasquali et M. Schönherr, Journal of Computational Physics, 348 (2017) 889-898. 
Après la visite de Prandtl accompagné par Theodore von Kármán à Auteuil en octobre 1913, Eiffel envoie les plans de son ventilateur hélicoïdal à Prandtl (fig. 4) qui modifie sa soufflerie à Göttingen en lui permettant d'augmenter la vitesse de $10 \mathrm{~m} / \mathrm{s}$ à $23 \mathrm{~m} / \mathrm{s}$, et ainsi de réaliser de nouvelles expériences qui confirment l'existence de la crise de trainée pour les sphères découverte par Eiffel [10].

C'est Prandtl qui, de plus, explique la phénoménologie de la transition observée à partir du changement de nature de la couche limite. Dans le régime sous-critique, celle-ci est laminaire et le tore de recirculation derrière le corps est généré par la séparation des lignes de courant à un angle de près de $90^{\circ}$ à partir du point de stagnation en amont (angle indiqué par des lignes en tirets dans les images de gauche de la figure 6). Dans le régime supercritique, l'angle de séparation augmente brusquement à près de $130^{\circ}$ (images de droite de la figure 6) ; ce changement s'accompagne d'une diminution notable de la taille de la recirculation, diminution déjà observée par Eiffel, et de trainée.

Nous nous trouvons donc en présence d'une couche limite turbulente qui peut être excitée prématurément par des rugosités ou des perturbations d'amplitude finie, ce qui réduit la valeur du nombre de Reynolds critique. Ce phénomène de crise de trainée est observé sur d'autres corps, et en particulier sur un long cylindre normal à l'écoulement avec des caractéristiques très similaires, tout en conservant un caractère bidimensionnel en moyenne.

Cette découverte, faite il y déjà plus d'un siècle par Gustave Eiffel sur le Champ de Mars, demeure, encore aujourd'hui, un sujet ouvert de la dynamique des fluides. Quoique l'existence de la crise de trainée soit bien établie, il reste à élucider sa dynamique intermittente et bistable, en explorant au-delà du comportement moyen les aspects spatiaux sous-jacents tels que des structures cohérentes instables et l'apparition de forces de portance. Rares sont encore les simulations numériques capables de monter à de très hauts nombres de Reynolds et qui permettraient d'apporter une réponse à ces questions. D'un point de vue expérimental, le contrôle des rugosités et des perturbations qui déclenchent la transition représente, de même, un défi à résoudre.

Nous remercions Eberhard Bodenschatz (Göttingen), Olivier Cadot (Liverpool), Michael Eckert (Munich), Etienne Guyon et Benoit Roman (Paris) pour leur aide et suggestions.
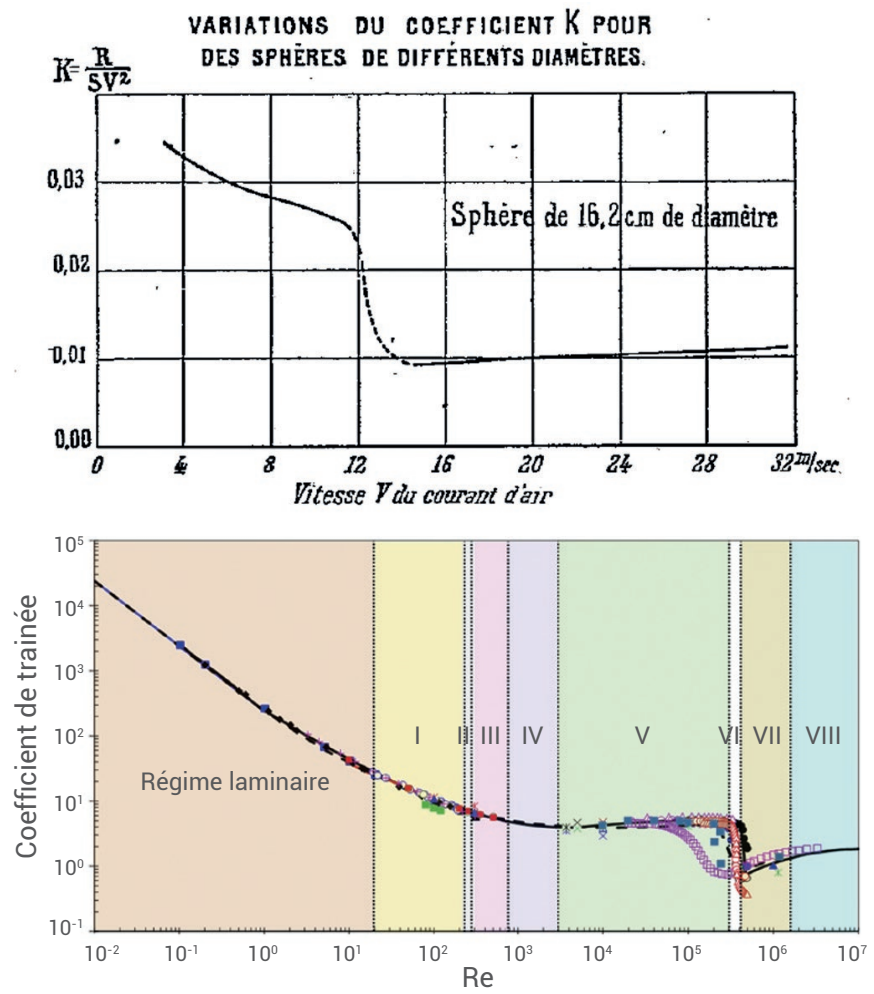

5. Mise en évidence de la « crise de trainée ». (a) Mesures par Eiffel du coefficient de trainée (défini dans la figure) d'une sphère de $16,2 \mathrm{~cm}$ de diamètre en fonction de la vitesse du vent : on note le changement de régime pour une vitesse critique d'environ $12 \mathrm{~m} / \mathrm{s}$. Cette vitesse critique diminue quand le diamètre de la sphère augmente [9]. (b) Compilation d'expériences récentes : variation du coefficient de trainée d'une sphère isolée en fonction du nombre de Reynolds Re, montrant l'existence de différents régimes d'écoulement [11].

a
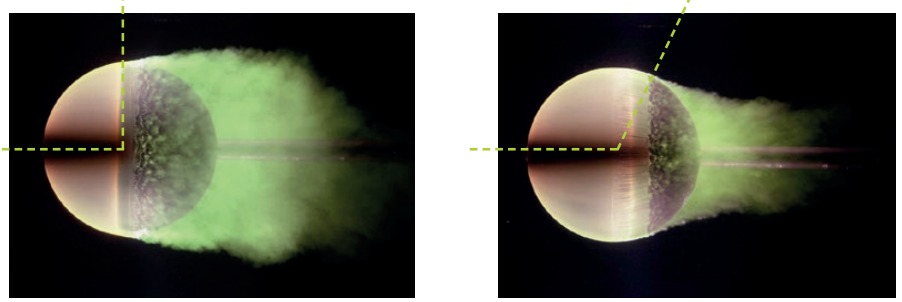

(b)
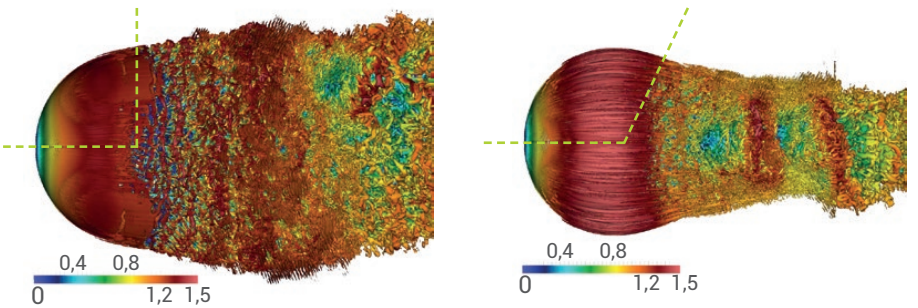

6. Modification de la turbulence autour d'une sphère soumise à un écoulement, en fonction du nombre de Reynolds. Les tirets verts indiquent l'angle de séparation des lignes de courant.

(a) Visualisation expérimentale en tunnel hydrodynamique de l'écoulement autour d'une sphère en régime laminaire (à gauche, $R e=200000$ ) et en régime turbulent (à droite, $R e=300000$ ). (Photographies par H. Werle, ONERA)

(b) Simulation numérique des structures instables [12] : contour des régions où la vorticité domine le cisaillement avant (à gauche, $\mathrm{Re}=100000$ ) et après (à droite, $\mathrm{Re}=1140000$ ) le régime critique. Les couleurs représentent la vitesse en unités de la vitesse d'entrée du fluide.

(a) Cette soufflerie que l'on peut visiter, située au 67 rue Boileau dans le $16^{\mathrm{e}}$ arrondissement de Paris, est classée monument historique et appartient actuellement au Centre Scientifique et Technique du Bâtiment (CSTB). Elle abrite également l'appareil de chute original. 